\title{
M2M Potentials in logistics and transportation industry
}

 \\ Anna Förster ${ }^{1} \cdot$ Yasir Zaki $^{3}$ - Carmelita Görg ${ }^{1}$ Andreas Timm-Giel ${ }^{4}$
}

Received: 15 December 2015/ Accepted: 16 July 2016/Published online: 28 July 2016

(c) The Author(s) 2016. This article is published with open access at Springerlink.com

\begin{abstract}
All over the world, road congestion is among the most prevalent transport challenges usually in urban environments which not only increases fuel consumption and emission of harmful gases, but also causes stress for the drivers. Intelligent Transportation System (ITS) enables a better use of the infrastructure by connecting vehicles to other vehicles as well as infrastructure and thus delivers a faster communication opportunity to ensure safe and secure driving. Machine-to-machine (M2M) communication is one of the latest information and communication technologies which offers ubiquitous connectivity among several smart devices. The use of mobile (cellular) M2M communications has emerged due to the wide range, high reliability, increased data rates, decreased costs as well as easy and short-term deployment opportunities. Since the radio spectrum is a scarce resource, M2M traffic can potentially degrade the performance of mobile networks due to the large number of devices sending small-sized data. This paper presents an efficient data multiplexing
\end{abstract}

This article is part of a focus collection on "Dynamics in Logistics: Digital Technologies and Related Management Methods."



scheme by using Long-Term Evolution Advanced (LTEAdvanced) Relay Nodes, which aggregates M2M traffic to maximize radio resource utilization. Extensive systemlevel simulations are performed using an LTE-Advancedbased model developed in the RIVERBED modeler to evaluate the performance of the proposed data multiplexing scheme. Simulation results show that approximately $40 \%$ more smart M2M devices used in ITS and logistics are served per LTE-Advanced cell under the present system settings.

Keywords Road congestion · Intelligent transportation systems - Information and communication technologies . Machine to machine - Relay nodes - LTE-Advanced

\section{Introduction}

The dramatic use of communication technologies (wired and wireless), embedded systems as well as increasing penetration of the Internet has not only revolutionalized human lives, but also reshaped almost all types of business

\footnotetext{
Andreas Timm-Giel timm-giel@tuhh.de

1 Communication Networks, University of Bremen, Bremen, Germany

2 Department of Computer Systems Engineering, University of Engineering and Technology, Peshawar, Peshawar, Pakistan

3 Computer Science Department, New York University Abu Dhabi (NYUAD), Abu Dhabi, UAE

4 Institute of Communication Networks, University of Technology, Hamburg, Germany
} 
models and processes [1]. M2M communication is one of the emerging technologies which offers ubiquitous connectivity among intelligent devices, hence is one of the major enablers of the Internet-of-Things (IoTs) vision [2]. IoT is an innovative concept which offers to connect smart devices often called things endowed with several sensing, automation as well as computing capabilities, with the Internet [3]. Resultantly, the connected devices are revolutionalizing the future cyber physical systems, yielding several applications. Moreover, the mobile network operators are partnering with industrial organizations in order to bring forth innovative IoT services to facilitate end consumers. For instance, M2M applications include intelligent transportations, logistics and supply chain management, e-health, smart metering, surveillance and security, smart cities, and home automation [4-6]. Thus, M2M communication is foreseen to reshape the business of operators, service providers, M2M enterprises, and M2M enablers [7].

Vodafone revealed that M2M communication is becoming one of the driving forces for businesses which inspires to bring forth innovative solutions almost in every sector such as logistics, automotive industry, cities, homes, schools, and workplaces [8]. Approximately $90 \%$ of the companies worldwide have adopted M2M technology and imparted it as one of the most favorable technologies for achieving noticeable outcomes. Automotive industry is one of the top sectors for adopting M2M technology. Approximately 32 and $17 \%$ increasing growth rates for adopting M2M technology have been noticed in automotive and logistic sectors, respectively, as shown in Fig. 1. Thus, ITS and logistics are considered as one of the potential M2M users worldwide [9]. In addition, NOKIA forecasted that the use of M2M technology in automotive industry and logistical processes will dominate other applications in the future [10]. One of the major motivations is to deliver a fully managed infrastructure which primarily guarantees, e.g., safe and secure driving, in time delivery, smart monitoring, and tracking of assets. Resultantly, this can revolutionize the existing methods of transportation and freight movements. In addition, optimum system performance can be achieved by reducing factor of costs, pollution and emission of harmful gases.

Mobile M2M communication greatly differs from traditional human-to-human $(\mathrm{H} 2 \mathrm{H})$ communication in terms of traffic density, data packet size, and quality of service (QoS) requirements [11]. For instance, an experimental study done in [12] shows that M2M traffic exhibits a significantly different behavior than the traditional smartphone traffic in various aspects. For example, unlike traditional mobile traffic, M2M is an uplink ${ }^{1}$ dominant traffic which particularly generates bursty traffic volumes.

$\overline{1}$ Sending information from M2M devices to base station.



Fig. 1 An illustration of leading industries adoption M2M technology worldwide, based on the Vodafone report 2015 [8]

Besides, it also exhibits unique characteristics such as an increasing device volume, sending small payloads, the demand for various mobility profiles, time-controlled, and mainly delay tolerant. Since the spectrum for mobile networks will remain a scarce resource, efficient utilization of radio spectrum is one of its major requirements. Therefore, the objective of this paper is to exploit LTE-Advanced RNs to multiplex small-sized M2M data packets in order to ensure efficient LTE-Advanced radio resource utilization and thus to support a large number of devices.

The rest of the paper is structured as follows. We firstly present an overview of the leading communication technologies used in ITS and logistics, followed by a generic overview of the two latest technologies, i.e., the Institute of Electrical and Electronics Engineers (IEEE) 802.11p and 3GPP LTE-Advanced in Sect. 2. An overview of major M2M services in ITS and logistics are discussed in Sect. 2. Then, we discuss problem definitions by highlighting how LTE-Advanced resources can be used inefficiently by smart M2M devices in Sect. 3. Section 4 presents the proposed uplink M2M data multiplexing scheme [13]. The simulation environment and parameter settings are presented in Sect. 5. We discuss our simulation results in Sect. 6. In the end, conclusions are drawn in Sect. 7. In addition, a list of most frequently used acronyms is presented in Table 1.

\section{Mobile M2M communications}

This section presents an overview of ETSI (European Telecommunications Standards Institute) M2M architecture followed by the major M2M use cases and services in transportation and logistics.

\subsection{ETSI M2M architectural overview}

The high-level ETSI M2M network architecture is shown in Fig. 2 [14]. The major components of mobile M2M communication architecture include the device, communication as well as server domains. The primary functionality of the device domain is to collect and send sensor data such 
Table 1 List of used abbreviations

\begin{tabular}{ll}
\hline Abbreviation & Acronym \\
\hline MAC & Medium Access Control \\
MME & Mobility Management Entity \\
PHY & Physical Layer \\
PRB & Physical Resource Block \\
QCI & QoS Class Identifiers \\
RLC & Radio Link Control \\
RRC & Radio Resource Control \\
SAE & System Architecture Evolution \\
S-GWs & Serving Gateways \\
SGSN & Serving GPRS Support Node \\
TTI & Transmission Time Interval \\
WLAN & Wireless Local Area Network \\
WPAN & Wireless Personal Area Network \\
WSN & Wireless Sensor Network \\
\hline
\end{tabular}

as the internal temperature and humidity level of a container, position and speed of a vehicle, and fuel consumption. The role of the communication network is to create a communication path between the devices and servers through either wired or wireless networks such as Digital Subscriber Line (DSL) and cellular networks (e.g., LTE-Advanced), respectively. Finally, the server domain consists of a middleware layer where the collected packets go through several application services and later are used by related agencies. Thus, M2M technology employs wired, wireless, and hybrid communication opportunities among devices to ensure a fully automatic acquisition, processing, and transmission of data. Thus, M2M represents a broad next-generation technology that is primarily incorporated in modern automobile industries to improve the ease, safety, and quality of human life. The wired access technologies provide less delay, high throughput and are more reliable. Despite the aforementioned capabilities, wired systems cannot be used in transport and logistical systems due to several limitations such as lack of scalability, cost efficiency, and mobility. However, abovementioned limitations can be overcome by incorporating wireless (cellular) technologies to achieve maximum system efficiency and reliability.

Recently, the key emerging wireless technologies used in modern transport and logistical systems include IEEE 802.11p [15] (standardized to support vehicular communication) and $3 \mathrm{GPP}^{2}$ LTE-Advanced mobile networks [16]. IEEE $802.11 \mathrm{p}$ is achieved by making few advancements in well-known 802.11 Wireless Local Area Network (WLAN) technology. It is also considered as the most feasible

\footnotetext{
2 3rd Generation Partnership Project-3GPP-is the leading standardization organization for mobile networks.
}

technology for Vehicular Ad hoc Networks (VANET). IEEE $802.11 \mathrm{p}$ wireless access introduces minimum delay in ITS. However, its employment is limited due to its decentralized nature. The recent research has revealed that maximum operating efficiency of IEEE $802.11 \mathrm{p}$ can be achieved by supporting it with the LTE-Advanced mobile networks. The main features of LTE-Advanced technology include wide availability of modules/devices, system existence, decreased costs as well as easy deployment. Since the cellular modules and sensors are easily available, e.g., in vehicles, the applications of LTE-Advanced mobile networks are dramatically increasing in automotive sector.

The authors in [16-18] compared the performance of two different technologies under varying channel and traffic load conditions. Moreover, several issues due to decentralized nature of IEEE $802.11 \mathrm{p}$ are highlighted in [16]. For instance, the main shortcomings are less reliability due to uncoordinated Medium Access Control (MAC) procedures, risk of network congestion, higher vehicle mobility, and low scalability. Moreover, the number of network-connected vehicles adversely affect the performance of the standard system. On the other hand, LTE-Advanced networks are more reliable, scalable, and capable of supporting higher density of vehicles. In addition, the authors analyzed the performance of the above technologies in terms of delay, reliability, scalability, and mobility support. The authors concluded that 3GPP LTEAdvanced systems are more efficient in terms of high scalability and mobility support than IEEE $802.11 \mathrm{p}$ standard. However, it is further concluded that the performance of IEEE $802.11 \mathrm{p}$ is more sensitive during increased density, traffic load, and higher mobility.

\subsection{M2M use cases}

Mobile M2M communication offers manifold applications and services in modern transport and logistical processes such as onboard security, traffic and infrastructure management, fleet management, and route planning [19]. In case of an emergency, the collected data are sent to other vehicles as well as infrastructure to gain immediate attention. To avoid further incidents, communication between the infrastructure and the vehicles must be very fast to detect emergency messages and deliver warning messages immediately. Similarly, traffic and infrastructure management play a prominent role in handling the problem of road congestion. It tackles the problem by providing two-way communication opportunities between vehicles and infrastructure. Vehicles can send status updates about the position, speed, fuel consumption, and delivery status reports to the infrastructure and can also receive relevant instructions about road accidents and emergency braking system [20]. Moreover, M2M communications support 


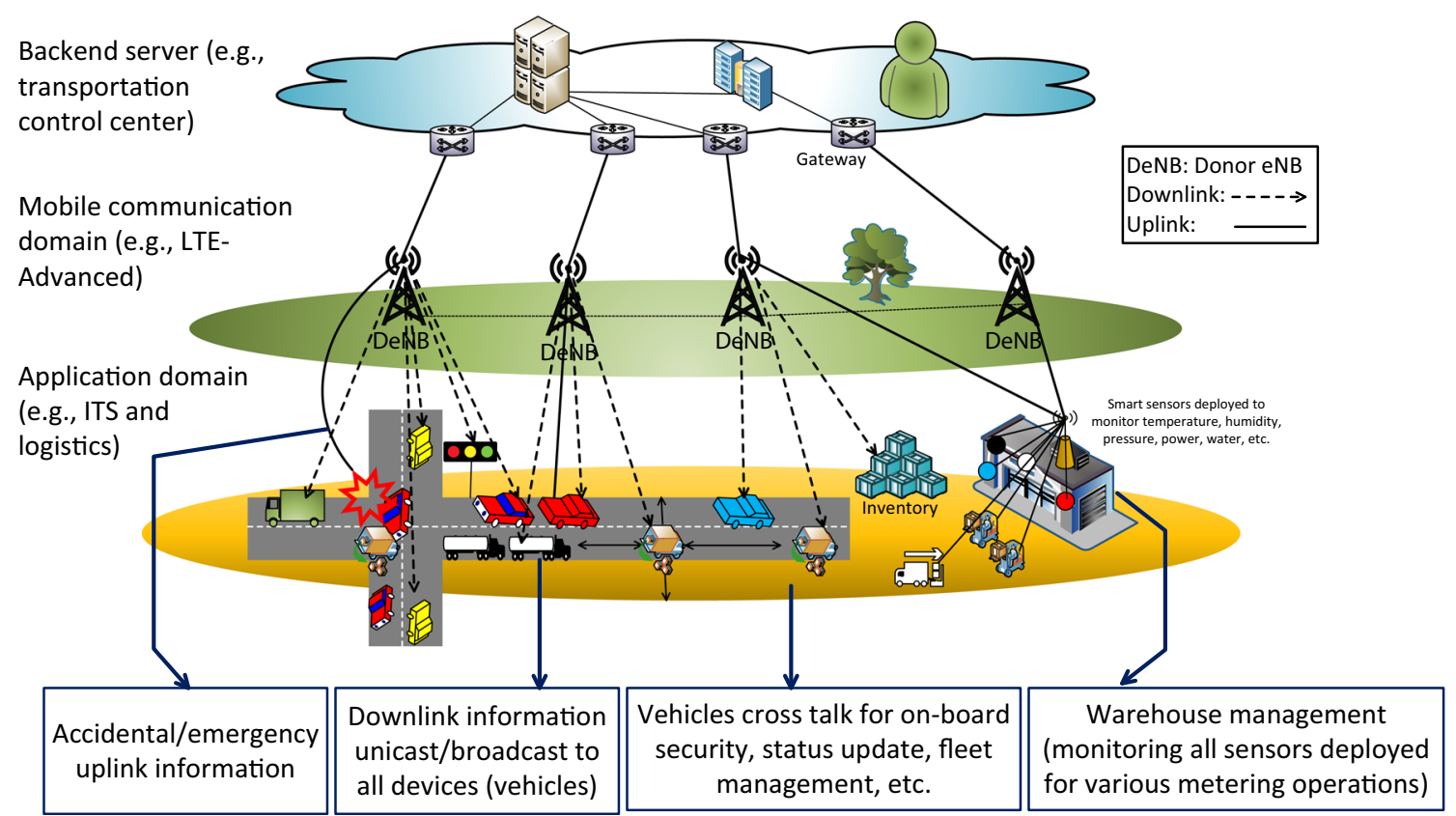

Fig. 2 ETSI mobile M2M communication architecture along with an overview of major M2M applications in intelligent transportation and logistics, based on [14]

several operations such as tracking of a stolen vehicle, traffic reports, and route planning as well as infotainment services [6]. For instance, to recover a stolen vehicle, SVT (Stolen Vehicle Tracking) service providers request data about the location from Telematic Control Unit (TCU) located inside the vehicle. In addition, drivers are also updated by sending reports regarding traffic in a particular region so that they can change or plan new routes in case of traffic jam or an emergency. Furthermore, infotainment services aim to provide news/information to drivers and passengers through mobile TV, web-browsing, etc.

Fleet management is also one of the major M2M applications in logistics [9]. The movements of vehicles, containers, buses, and cars are being tracked regularly through devices which collect data of the location, vehicle speed, temperature, distribution progress, fuel consumption and send this information to monitoring servers. Through regular monitoring, several activities of the system can be performed in an efficient way. For instance, the goods which are transported from one place to another are monitored regularly in order to accomplish in time delivery and to handle any undesirable situation during shipment processes. The cargo moves across several regions; therefore, it must be monitored in order to stay updated. Moreover, reporting gives the exact location of the freight, and thus the conditions of the objects can be easily monitored. In applications such as warehouse management, fleet management, robotics, and control systems, alarms are also used to detect critical or emergency situations.
In addition to the above applications, M2M communication provides additional services in logistics such as decreased operational cost, high inventory flexibility, increased supply chain visibility, and reduced loss of vehicles and containers [21]. In supply chains, M2M technology enables tracking the status of goods in real-time via M2M devices. This increasing visibility allows for significant reduction in uncertainties in supply chain [22]. Similarly, in a warehouse, M2M devices can be deployed to track the inventory so that stockholders and enterprises can respond to the market dynamics and to decide when to refill and when to go on sale. Moreover, cross talk among vehicles can also be effective to get immediate assistance. Additionally, direct delivery of inventory from one vehicle to another without storing it in a warehouse can also be accomplished through mutual information sharing. Consequently, it can significantly reduce the required space of warehouse, customer's waiting time as well as the operational costs for business entities.

\section{Problem descriptions}

Mobile systems such as LTE-Advanced are mainly designed to support the increasing traditional mobile traffic by enabling improved broadband services [23]. For instance, Ericsson in its mobility report [24] anticipates approximately 5.6 billion cellular-based active smartphones by 2019. Therefore, in order to support such a 




Fig. 3 An example illustration of inefficient PRB utilization for M2M communication

massive mobile traffic, several enhancements in existing LTE systems [25] such as Relay Nodes (RNs) [26], massive Multiple Input-Multiple Output (MIMO) systems [27], and femto cells [28] were introduced to fulfill the IMT-Advanced (International Mobile Telecommunications Advanced) demands of maximum throughput of upto 1 Gbps in downlink and $500 \mathrm{Mbps}$ in uplink [29]. On the other hand, mobile standards are foreseen as an attractive option to support future M2M applications such as in transportation, logistics, smart city, and living [2]. Several forecasts have also reported a considerable market growth for M2M device volume, making quality of service provisioning a huge challenge [10]. Consequently, mobile networks will not be able to support the increasing number of M2M devices worldwide [30].

According to $3 \mathrm{GPP}$, the smallest unit of the radio spectrum allocatable to a single device is $1 \mathrm{PRB}^{3}$ which is capable of transmitting several hundred bits under favorable channel conditions. However, allocating 1 PRB to a single M2M device used in transportation/logistics could significantly degrade radio spectrum utilization $[13,19,31]$. This is due to the fact that the capacity of a PRB can be much higher than the actual size of the device payload under favorable channel conditions. Figure 3 illustrates how inefficiently a PRB can be utilized when a device sends small-sized packets when experiencing good channel conditions. An exemplary device payload of 4 bytes is considered in order to evaluate the utilization of a PRB against all the possible values of the Modulation and Coding Scheme (MCS). A PRB utilization efficiency is evaluated in terms of percentage of the actual payload and the padded zeros with respect to MCS index. It can be seen from Fig. 3 that with the lower MCS values depicting unfavorable channel conditions, the PRB capacity is smaller, and therefore, it is fully utilized to transmit the given payload. On the other hand, with the higher MCS

\footnotetext{
${ }^{3}$ Physical Resource Block.
}

values, e.g., 20-26 representing favorable channel conditions, the capacity of the PRB increases. However, small packets with increasing percentage of padded zeros are transmitted. This shows that more packets can be accommodated per PRB under favorable channel conditions in the given exemplary scenarios. Since mobile radio resources are valuable assets and scarcely available, it is required to ensure efficient utilization of radio resources for M2M communications.

\section{Proposed M2M data multiplexing}

In the proposed data multiplexing scheme, the small-sized M2M data packets are multiplexed at the Packet Data Convergence Protocol (PDCP) layer of the RN. A highlevel illustration of E-UTRAN (Evolved UMTS Terrestrial Radio Access Network) architecture with the functionalities of LTE-Advanced relaying is depicted in Fig. 4. The RN communicates with devices using access link (Uu), whereas it communicates with DeNB using backhaul link (Un). Thus, it behaves like an eNB toward the M2M devices, whereas it acts as a UE toward the DeNB.

The RN PDCP is selected to aggregate M2M packets in order to maximize the multiplexing gain. This increasing multiplexing gain is achieved without aggregating the additional overheads such as those from the PDCP, Radio Link Control (RLC), and Medium Access Control (MAC). A high-level illustration of the proposed scheme is given in Fig. 5. Generally, the PDCP layer is a part of LTE air interface control and user plane protocols. It exists in the $\mathrm{UE}$, Donor eNB (DeNB), and in the RN. The major functionalities of PDCP layer for the user plane include header compression and decompression, user data transfer, delivery of upper layer Packet Data Units (PDUs) in sequence, and retransmission of PDCP Service Data Units (SDUs). Moreover, the control plane services include ciphering and integrity protection and transfer of control plane data. The proposed multiplexing scheme can also be applied at the lower layers of the RN such as RLC and MAC on the Uu interface or in the PDCP of Un interface. However, in these cases, the multiplexing gain will be reduced due to the multiplexing of additional headers along with the original small-sized data packets [13].

A multiplexing buffer is created at the RN PDCP layer which multiplexes the incoming packets according to the available service rate which is equal to Transport Block Size (TBS) - RN Un protocol overheads in terms of bits per TTI, as shown in Fig. 5. The Un protocol overhead includes 12, 28, and 4 bytes for GTP (GPRS Tunneling Protocol), UDP (User Datagram Protocol)/IP, and layer 2, respectively. The multiplexed packet is sent to the RN GTP over the Un link. The additional overheads such as from 


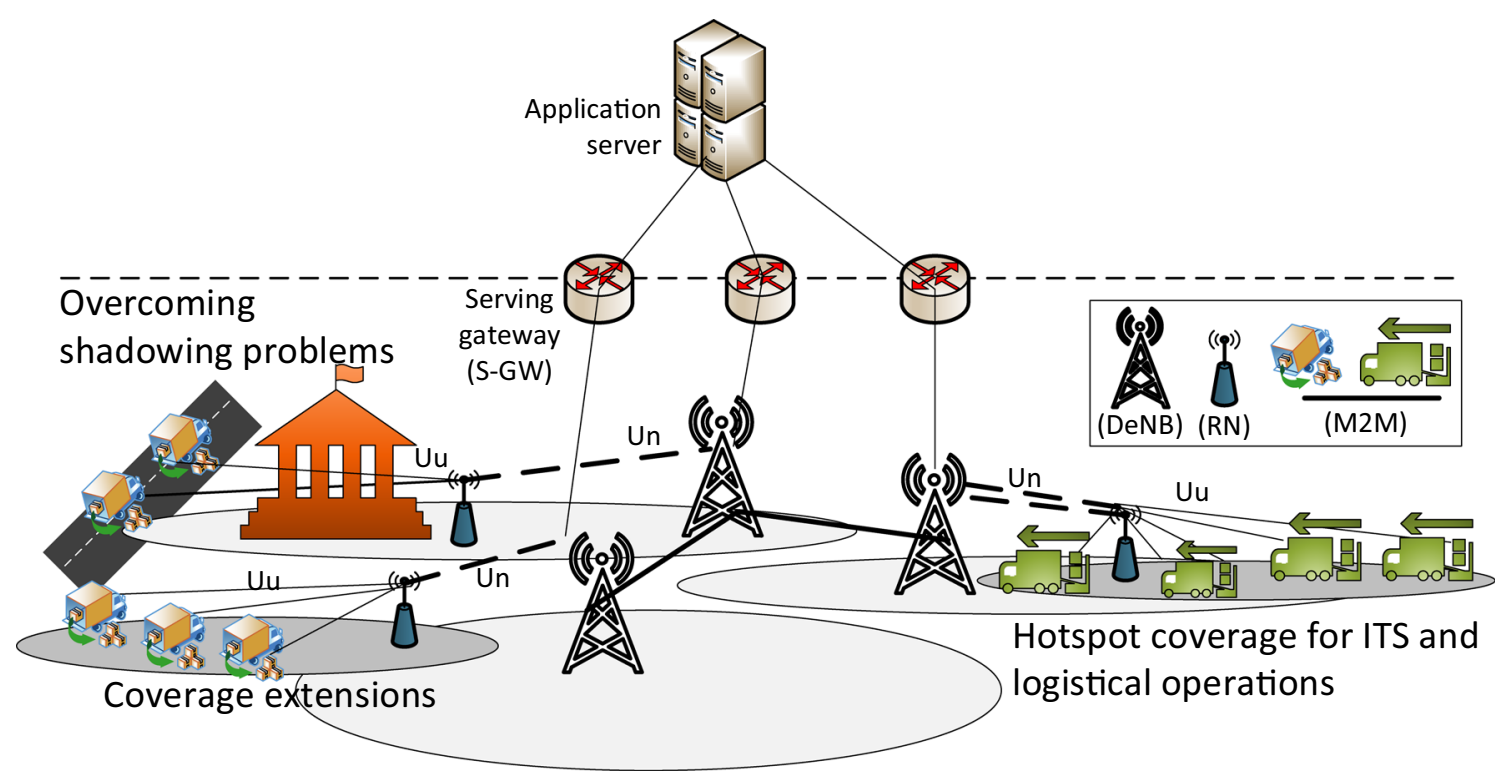

Fig. 4 An illustration of LTE-Advanced relaying technology. Re-drawn based on [26])

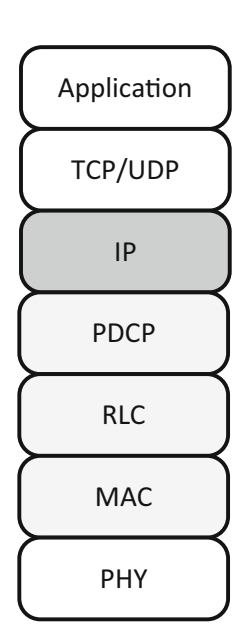

M2M device

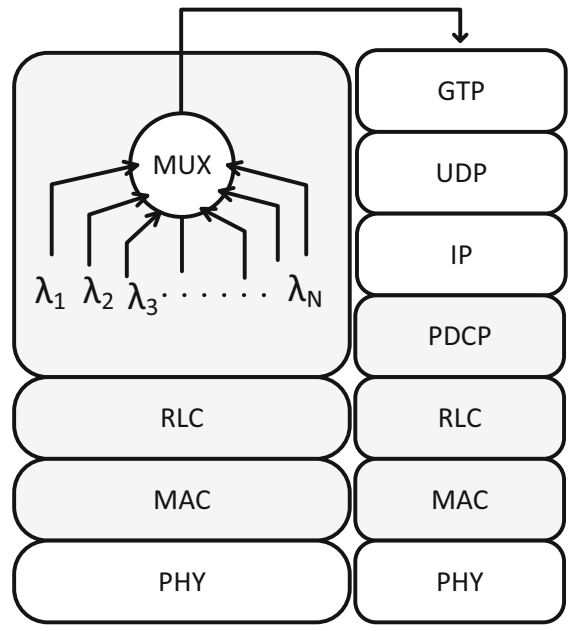

$\mathrm{RN}$

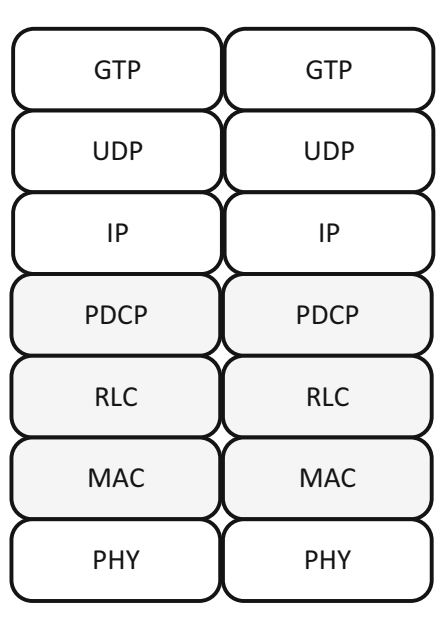

DeNB

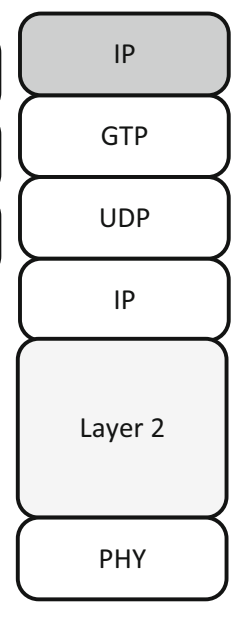

aGW

Fig. 5 A high-level illustration of the proposed multiplexing scheme along with protocols stack of the M2M device, RN, and DeNB

the GTP, UDP/IP, PDCP, and RLC are added. The block diagram of the data differentiating algorithm at the DeNB is given in Fig. 6. From the physical layer of the RN, the multiplexed packets are sent to the DeNB over the Un interface, as depicted in Fig. 5.

The multiplexing approach significantly improves PRB utilization. However, there are certain constraints regarding latency requirements of high priority M2M traffic such as accidental information, emergency alerting, and e-health. This is due to the fact that each packet waits until the size of the buffer B is equal to the available TBS-RN Un protocol overheads. In a highly loaded scenario, the waiting time is not long due to a high arrival rate. However, in a low loaded scenario, there is a comparatively longer delay due to the low arrival rate. Consequently, the performance of delay sensitive M2M applications can be degraded. To tackle this issue, an expiry timer, $T_{\max }$ is introduced. The timer is set with a fixed value such as $10 \mathrm{~ms}$ in the current implementation. This means that the buffer serves the multiplexed packet after $10 \mathrm{~ms}$ at the latest. The value of the timer could also be adaptive, i.e., it can change its value according to priorities of the incoming packets. For this purpose, the algorithm must be fully aware of various priorities of M2M applications.

In this work, a fixed $\mathrm{RN}$ is implemented and used to multiplex the uplink traffic from M2M devices deployed 


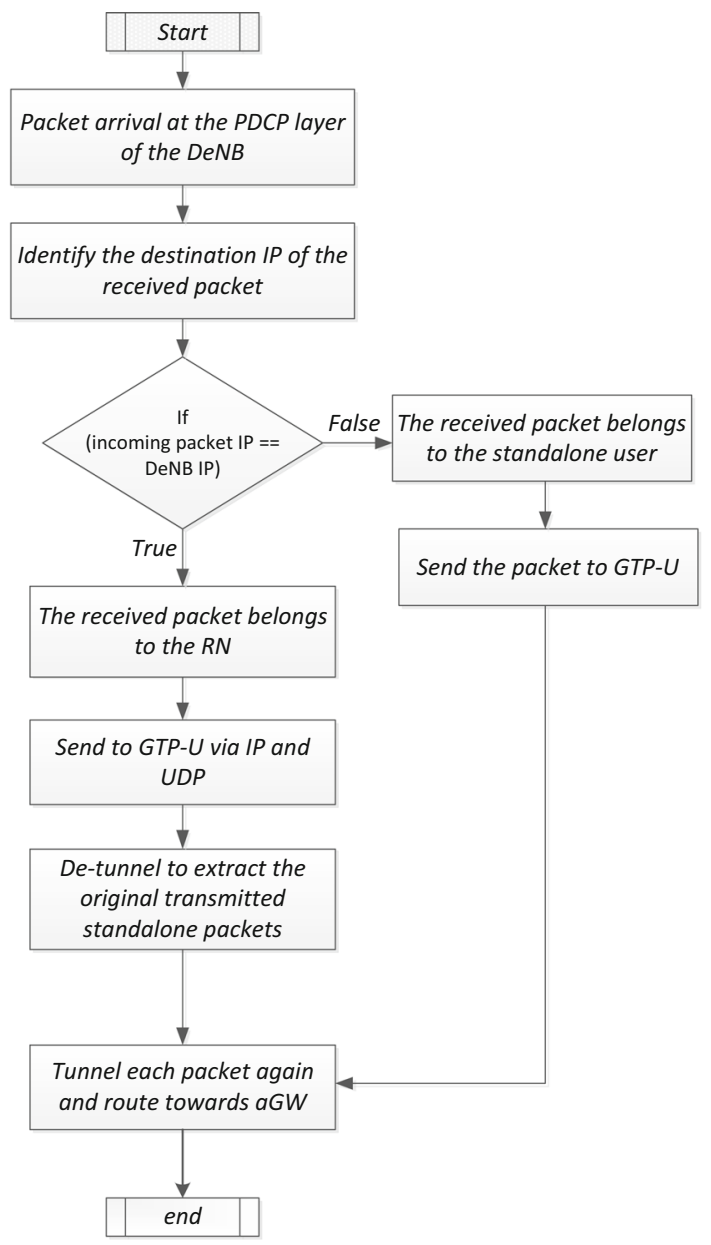

Fig. 6 Block diagram of the data differentiating algorithm at the DeNB

in ITS and logistics (i.e., for onboard security and metering purposes). The position of the RN corresponds to an MCS of 16, whereas 5 PRBs are allocated to RN to serve M2M traffic. The given values of MCS and PRBs correspond to TB size of 1608 bits per TTI. Since a TTI is of 1-ms duration, the TB size can also be given as $1608000 \mathrm{bits} / \mathrm{s}$. According to the 3GPP standardizations, the capacity of a single PRB varies according to the MCS. Therefore, under the given values of MCS and PRBs, 321 bits per TTI or 321000 bits/s can be sent within a single PRB. In general, the system utilization, $\rho$ can be determined in terms of the arrival rate, $\lambda$ and the service rate, $\mu$. Additionally, the maximum number of served devices, $N_{\max }$ can be determined according to the following procedure,

- The size of the each transmitted M2M packet per second is $P_{\text {size }}=656$ bits.

- The arrival rate $\lambda$ due to $N$ number of devices is $(N \times 656) b / s$.

- The service rate per PRB is $\mu_{\mathrm{PRB}}=321000 \mathrm{~b} / \mathrm{s}$.

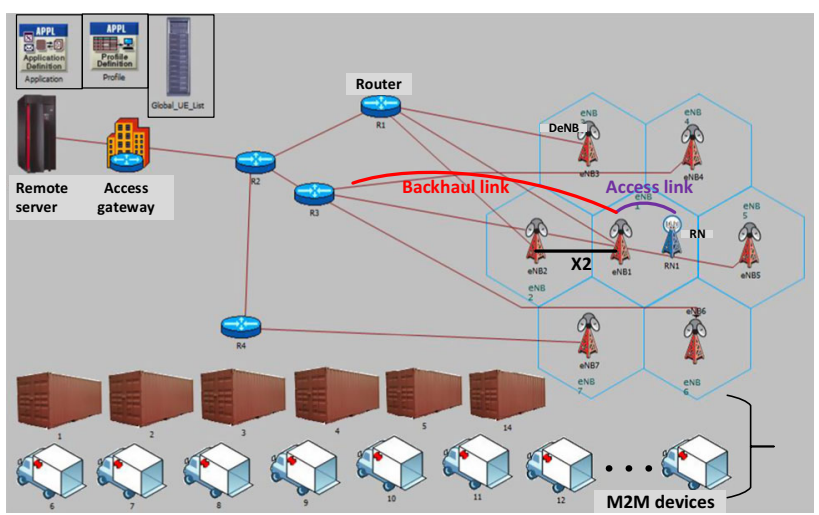

Fig. 7 Project editor of the LTE-Advanced-based model developed in the RIVERBED Modeler simulator

- The overall service rate is $\mu=\mu_{\mathrm{PRB}} \times 5$ (PRBs) b/s.

- So, the system utilization $\rho$ can be given as

$$
\begin{aligned}
& \text { Utilization, } \quad \rho=\frac{\text { Arrival rate }}{\text { Service rate }}=\frac{\lambda}{\mu} \\
& \text { Utilization, } \quad \rho=\frac{N \times 656}{321600}
\end{aligned}
$$

- Moreover, the maximum number of devices served can be given as follows,

$N_{\max }=\frac{\rho \times 321600}{656}$

\section{Simulation model and parameters}

The Optimized Network Engineering Tool (OPNET) Modeler which is newly named as RIVERBED Modeler is used as a primary modeling, simulation, and analysis tool for this research. It provides a simulation environment for the performance measurements of communication networks [32]. The project editor of the LTE-Advanced-based model developed in the simulator with several nodes along with LTE-Advanced functionalities and protocols is shown in Fig. 7. Additionally, an RN is implemented and placed within the coverage of DeNB to relay and multiplex M2M data traffic. The DeNB is responsible to connect UEs/M2M devices with the transport network, thus includes both radio interface and transport protocols to communicate with UEs/M2M devices and the core network, respectively. The radio protocols traffic coming from aGW toward the RN. The remote server and the aGW (access gateway) are interconnected with an Ethernet link with an average delay of $20 \mathrm{~ms}$. Moreover, aGW acts as the tunneling point for the downlink and uplink traffic coming from PDN-GW and DeNB, respectively. The aGW node protocols include the 
Table 2 Simulation parameters

\begin{tabular}{|c|c|}
\hline Parameters & Values \\
\hline Simulation length & $1000 \mathrm{~s}$ \\
\hline eNB coverage radius & $350 \mathrm{~m}$ \\
\hline Min. eNB UE distance & $35 \mathrm{~m}$ \\
\hline Max terminal power & $23 \mathrm{dBm}$ \\
\hline Terminal speed & $120 \mathrm{~km} / \mathrm{h}$ \\
\hline Mobility model & Random way point (RWP) \\
\hline Frequency reuse factor & 1 \\
\hline Transmission bandwidth & $5 \mathrm{MHz}$ \\
\hline No. of PRBs & 25 \\
\hline MCS & QPSK, 16QAM, 64QAM, \\
\hline Channel models & Pathloss, slow fading, fast fading \\
\hline Path loss & $128.1+37.6 \log _{10}(R), \mathrm{R}$ in $\mathrm{km}$ \\
\hline Slow fading & $\begin{array}{l}\text { Log-normal shadowing, correlation } \\
1 \text {, deviation } 8 \mathrm{~dB}\end{array}$ \\
\hline Fast fading & Jakes-like method [33] \\
\hline \multicolumn{2}{|l|}{$R N$ parameters } \\
\hline PRBs for RN & 5 \\
\hline Corresponding MCS & 16 \\
\hline TBS & 1608 bits \\
\hline Simulated scenarios & $\begin{array}{l}\text { No multiplexing, multiplexing } \\
\text { without timer, and multiplexing } \\
\text { with timer }\end{array}$ \\
\hline Timer expiry values & $10 \mathrm{~ms}$ (Sect. 6.2) \\
\hline Timer expiry values & 5, 10 and $20 \mathrm{~ms}$ (Sect. 6.3) \\
\hline Type of RN & Fixed \\
\hline \multicolumn{2}{|l|}{ M2M Traffic model [34] } \\
\hline Message size & 38 bytes (constant) at the device PDCP \\
\hline Inter-send time & $1 \mathrm{~s}($ constant $)$ \\
\hline
\end{tabular}

Internet Protocol (IP) and Ethernet. The aGW and DeNB nodes (names as eNB1..) communicate through IP routers (R1..). QoS parameters at the Transport Network (TN) ensure QoS parameterization and traffic differentiation. The user movement in a cell is emulated by the mobility model by periodically updating the location of the user. The user mobility information is stored in the global user database (Global-UE-List). The channel model parameters for the air interface include path loss, slow fading, and fast fading models. In this paper, the simulation modeling mainly focuses on the user plane to perform E2E performance evaluations. Furthermore, Table 2 presents a detailed description of simulation parameters and settings.

\section{Results and discussion}

This section investigates the performance of the proposed multiplexing scheme in the low and high loaded scenarios. We firstly describe all scenarios which are simulated in this work. Later, we discuss our simulation results in detail with varying load conditions. In this work, we consider M2M devices used in ITS and logistics in order to generate uplink traffic.

\subsection{Simulation scenarios description}

The scenarios are simulated according to three major categories, i.e., no multiplexing, multiplexing without timer, and multiplexing with timer. In the first category, M2M data packets are relayed in uplink without multiplexing. In the second category, the data packets from all the active devices are multiplexed at the RN before being sent to the DeNB. However, in this category, no expiry timer is considered to control the multiplexing process, and the multiplexed packet is served when its size is large enough to utilize full capacity of the available spectrum. In the third category, an expiry timer is introduced in order to limit the multiplexing delay especially in the low loaded scenarios. In this case, the multiplexed packet is served after $T_{\max }$ at the latest. Each category is further divided into several scenarios. The scenarios are simulated for both load conditions (i.e., low and high). In the low loads, the number of devices, $N$, is kept 200 in the first scenario. The value of $N$ is incremented by 200 in the subsequent scenarios till the limiting case, i.e., when all 5 PRBs are fully utilized. In high loads, $2800^{4}$ devices are placed in the first scenario, and the number is also incremented by 200 in the subsequent scenarios.

\subsection{Simulation results}

The simulation results for the mean number of used PRBs with $95 \%$ confidence interval (CI) are illustrated in Figs. 8 and 9 for the low and high load conditions, respectively. The values of the upper and lower bound of CI are very small in most of the scenarios as shown in Figs. 8 and 9. These PRBs are used by RN to transmit the multiplexed data toward the DeNB.

The simulation results clearly show the efficient utilization of PRBs in uplink with the proposed multiplexing scheme. For instance, in the no multiplexing scenario with 400 devices, the arrival rate at the PHY layer of the $\mathrm{RN}$ is $262.4 \mathrm{bit} / \mathrm{TTI}$ which almost utilize 1 PRB. However, in the case of multiplexing, only half of the PRBs are used to serve the 400 devices, see Fig. 8. Similarly, without multiplexing, the RN serves nearly 2400 devices with 5 PRBs in uplink, which is actually the limiting case as shown in

\footnotetext{
${ }^{4} 5$ PRBs are allocated to RN in order to serve M2M devices. These PRBs are fully utilized when the number of devices is raised to 2600 without multiplexing. So the high load scenarios start with 2800 devices.
} 




Fig. 8 A comparison of mean PRBs used with and without data multiplexing in low loaded scenarios. $T_{\max }=10 \mathrm{~ms}$

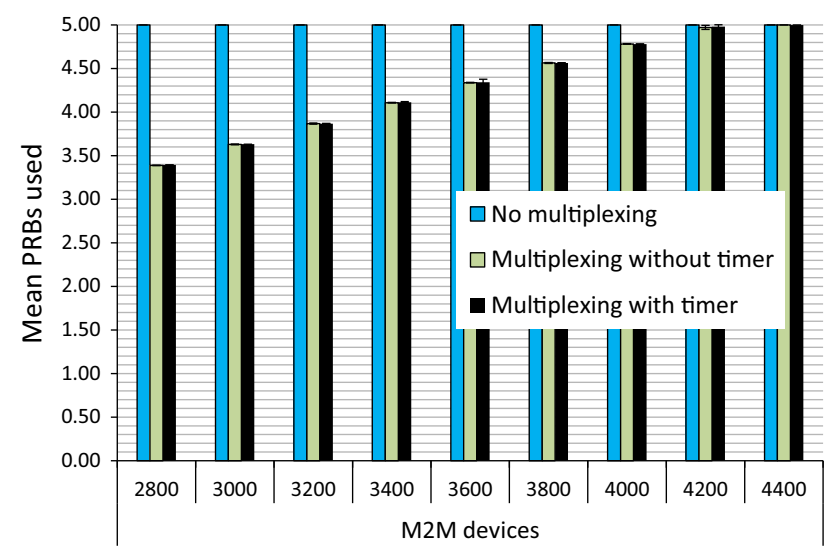

Fig. 9 Comparing simulation results of mean PRBs used with and without data traffic multiplexing in high loaded scenarios. $T_{\max }=10 \mathrm{~ms}$

Fig. 8. However, in case of multiplexing, the number of devices served by the RN nearly doubles, i.e., approximately 4200 devices are served as depicted in Fig. 9. This is due to the fact that in the case of no multiplexing, each data packet contains an additional Un air interface overhead of GTP, UDP, IP, and layer 2. The additional overhead causes an extra PRB usage and overall reduces the PRB utilization efficiency. Moreover, in low loaded scenarios such as with 200 devices (see Fig. 8), the average number of PRBs used is slightly higher in the case of multiplexing with timer. This is due to the fact that the RN serves the traffic at the latest after $10 \mathrm{~ms}$, and thus, the PRB is not necessarily used with its maximum capacity due to the low arrival rate. However, in high load scenarios, the timer has almost no impact, and a nearly equal number of PRBs is used with and without timer.

Figures 10 and 11 show the simulation results of the mean packet E2E delay in low and high load scenarios, respectively. The results show that the value of packet E2E

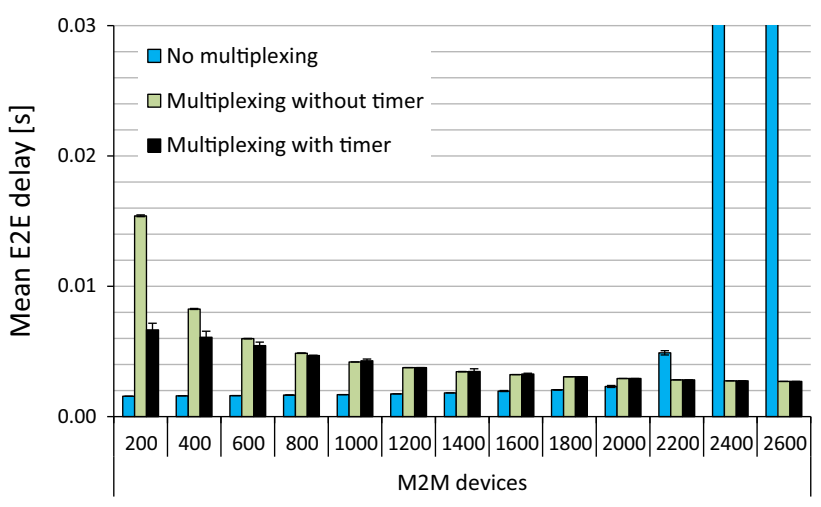

Fig. 10 A comparison of packet mean E2E delay in low loaded scenarios. $T_{\max }=10 \mathrm{~ms}$

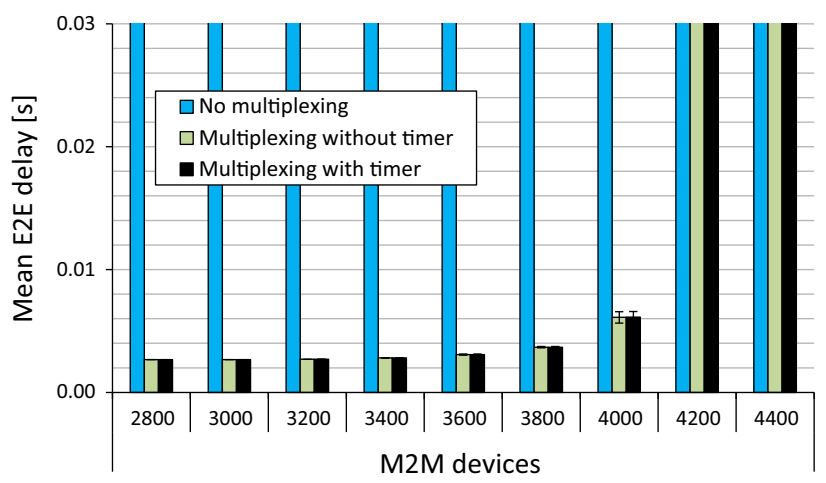

Fig. 11 Comparing packet mean E2E delay in high loaded scenarios with timer expiry, $T_{\max }=10 \mathrm{~ms}$

delay is higher in the case of multiplexing for low loads as shown in Fig. 10. This increase in delay time is due to less arriving packets especially in the low loaded scenarios. The buffer aggregates packets until its size plus the additional Un overhead is equal to the available TBS. However, the use of an expiry timer limits the delay by serving the aggregated packets at the latest after $10 \mathrm{~ms}$. On the other hand, in high loaded scenarios, the E2E delay is slightly higher compared to the case of no multiplexing, see Fig. 11. This is due to high arrival rate and the buffer aggregates the incoming packets to make a large aggregated packet within less time. Moreover, the value of the E2E delay is very large in fully loaded scenarios as depicted in Fig. 10, when the RN utilizes all 5 PRBs with the maximum capacity.

\subsection{Impact of timer expiry on data multiplexing}

In this section, we investigate the impact of timer expiry, $T_{\max }$ on mean PRB utilization and E2E delay by varying input traffic. However, we only consider low loaded scenarios due to the fact that the timer expiry has almost no 




Fig. 12 A comparison of packets mean E2E delay

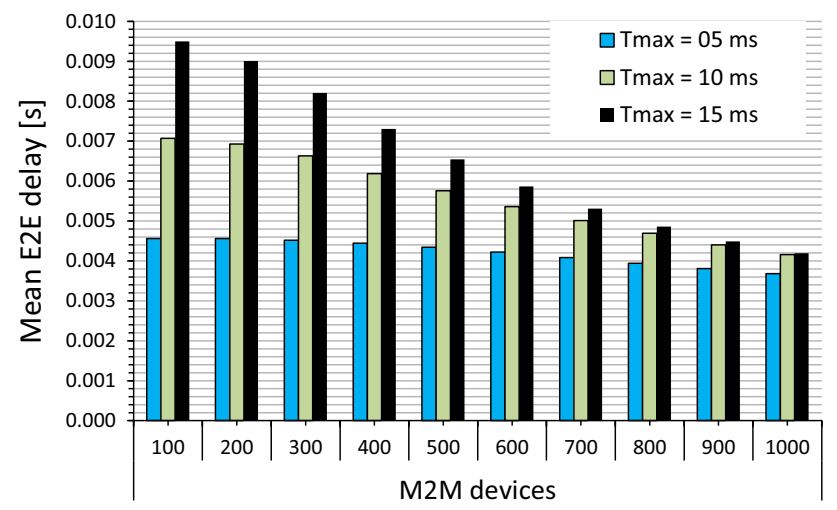

Fig. 13 A comparison of packets mean E2E delay

affect on PRB utilization and mean E2E delay in high loaded scenarios in the multiplexing process, as discussed earlier in Figs. 10 and 11. In general, the mean value of used PRBs decreases and E2E delay increases for larger values of the timer expiry. Additionally, this trade-off is heavily dependent on the arrival rate, as discussed earlier.

In this work, 10 scenarios are simulated in order to evaluate the impact of timer expiry on the multiplexing process. In the first scenario, $100 \mathrm{M} 2 \mathrm{M}$ devices are considered to generate input traffic. The number of devices is incremented by 100 in the subsequent scenarios. The above scenarios are simulated for the timer expiry values of 5, 10, and $15 \mathrm{~ms}$. The simulation results of mean number of used PRBs and E2E delay are compared for the given values of maximum waiting time. Figures 12 and 13 compare the simulation results of mean PRB utilization and E2E delay, respectively. Figure 12 shows that less PRBs are used in the case of larger values of $T_{\max }$, when the arrival rate is kept constant. For instance, in the first scenario with $N=100$, average numbers of PRBs used are 0.48, 0.27, and 0.20 for the timer expiry values of 5,10 , and $15 \mathrm{~ms}$, respectively. It shows that for the larger values of maximum waiting time, less number of PRBs is used on average, as it allows more packets to be multiplexed before timer expiry. Resultantly, it increases the multiplexing gain. Additionally, it is further noted that larger values of $T_{\max }$ has almost no affect on PRBs used in the case of high loads. For instance, when $N=1000$, the average values of PRBs used are 1.219 and 1.213 for the maximum waiting time of 10 and $15 \mathrm{~ms}$, respectively.

Similarly, Fig. 13 compares the simulation results of the mean E2E delay for the given timer expiry values. As discussed in the beginning, the larger values of waiting time, $T_{\max }$, increase the mean packet E2E delay, particularly in the low loaded scenarios. However, the effect reduces with the larger values of $N$. For instance, in the low loaded scenario when $N=100$, the given values of maximum waiting time introduce a mean E2E delay of $0.0045,0.0070$, and $0.0095 \mathrm{~ms}$, respectively. Since the effect of timer on the multiplexing process decreases due to the increasing arrival rate, the mean E2E delay values are reduced to $0.00368,0.00416$, and 0.00419 , respectively, for $N=1000$. Furthermore, it is noted that the values of mean E2E delay for $T_{\max }$ of 10 and $15 \mathrm{~ms}$ are almost similar. Moreover, the impact of timer expiry completely vanishes when input traffic load is increased beyond 1000, as discussed earlier in Figs. 10 and 11.

\section{Conclusion}

The latest 3GPP LTE-Advanced networks have primarily focused on dominating non-cellular technologies to support M2M applications also in the future. Since automotive applications and logistical processes demand wide coverages, increased data rates, high reliability, and low costs, LTE-Advanced is considered as the ready-to-use technology to fulfill the future M2M service requirements. Nevertheless, the major challenge is to support the myriad of devices sending small-sized data and thus inefficiently utilizing radio resources (PRBs). Since the spectrum for mobile networks will remain a scarce resource, new concepts for new traffic type demand careful planning and evaluation. In this paper, the data multiplexing scheme is proposed to overcome the risk of inefficient PRB utilization for mobile M2M traffic. Our results show that approximately $40 \%$ more M2M devices in ITS and logistics are served under the present system settings as compared to the state of the art without the use of multiplexing approach. Additionally, the QoS provisioning of the M2M traffic is ensured by considering an expiry timer which minimizes the multiplexing delays especially in low loaded scenarios. More importantly, approximately $60 \%$ of PRB utilization is improved for a maximum timer expiry value of $15 \mathrm{~ms}$ especially in low loaded scenarios. Thus, the proposed scheme can dramatically reduce the network 
load by sharing PRBs among several devices and can be a very useful scheme for network operators as well as service providers.

\section{Compliance with ethical standards}

Conflict of interest The authors have no conflict of interest in this manuscript.

Open Access This article is distributed under the terms of the Creative Commons Attribution 4.0 International License (http://crea tivecommons.org/licenses/by/4.0/), which permits unrestricted use, distribution, and reproduction in any medium, provided you give appropriate credit to the original author(s) and the source, provide a link to the Creative Commons license, and indicate if changes were made.

\section{References}

1. Zhang Y, Yu R, Nekovee M, Liu Y, Xie S, Gjessing S (2012) Cognitive machine-to-machine communications: visions and potentials for the smart grid. IEEE Netw 26(3):6-13

2. Taleb T, Kunz A (2012) Machine type communications in 3GPP networks: potential, challenges, and solutions. IEEE Commun Mag 50(3):178-184

3. Atzori L, Iera A, Morabito G (2010) The internet of things: a survey. Comput Netw 54(15):2787-2805

4. Exalted: Expanding LTE for Devices. http://www.ict-exalted.eu/ fileadmin/documents/EXALTED_WP2_D2.1.pdf. Accessed 30 Sept 2013

5. Towards 50 billion connected devices. Technical report, ERICSSON (2010)

6. ETSI: Machine-to-Machine communications (M2M); Use Cases of automotive applications. In: M2M capable networks. Technical report, TR 102898 V1.1.1 (2013)

7. Slicing up the M2M revenue pie; How to get your share and boost your business. Technical report, COMARCH (white paper)

8. Vodafone M2M Adoption Barometer. Technical report, Vodafone (2015)

9. The state of $\mathrm{M} 2 \mathrm{M}$ in transport and logistics; Key findings from the 2013 Vodafone M2M Adoption Barometer. Technical report, Vodafone (2014)

10. NOKIA: LTE M2M: optimizing LTE for the internet of things. Technical report, Nokia white paper (2014)

11. Laner M, Svoboda P, Nikaein N, Rupp M (2013) Traffic models for machine type communications. In: Proceedings of the tenth international symposium on wireless communication systems (ISWCS). VDE, pp 1-5

12. Shafiq MZ, Ji L, Liu AX, Pang J, Wang J (2012) A first look at cellular machine-to-machine traffic: large scale measurement and characterization. In: ACM SIGMETRICS performance evaluation review, vol 40. ACM, pp 65-76

13. Mehmood Y, Nawaz Khan Marwat S, Grg C, Zaki Y, Timm-Giel A (2015) Evaluation of M2M data traffic aggregation in LTE-A uplink. In: ITG/VDE mobile communication conference. VDE, pp 24-29

14. ETSI: Machine-to-Machine communications (M2M), Functional architecture. Technical report, ETSI TS 102690 V2.1.1 (2013)

15. IEEE, Wireless Access in Vehicular Environments. http://stan dards.iee.org/findstds/standard/802.11p-2010.html. Page Accessed 21 Nov 2015
16. Mir ZH, Filali F (2014) Lte and ieee 802.11 p for vehicular networking: a performance evaluation. EURASIP J Wirel Commun Netw 2014(1):1-15

17. Chou CM, Li CY, Chien WM, Lan KC (2009) A feasibility study on vehicle-to-infrastructure communication: Wifi vs. wimax. In: Tenth international conference on mobile data management: systems, services and middleware, 2009. MDM'09. IEEE, pp 397-398

18. Trichias K, Berg VDJ, Heijenk G, Jongh DJ, Litjens R (2012) Modeling and evaluation of LTE in intelligent transportation systems. In: Joint ERCIM eMobility and MobiSense Workshop, Santorini, Greece, pp 48-59

19. Mehmood Y, Goerg C, Muehleisen M, Timm-Giel A (2015) Mobile M2M communication architectures, upcoming challenges, applications, and future directions. EURASIP J Wirel Commun Netw 2015:1-37

20. Greenwood DA, Dannegger C, Dorer K, Calisti M (2009) Dynamic dispatching and transport optimization-real-world experience with perspectives on pervasive technology integration. In: HICSS, pp 1-9

21. Machine to Machine (M2M)—Deutsche Telekom; Transport and Logistics. https://m2m.telekom.com/vertical-industries/transportand-logistics. Accessed 1 Sept 2015

22. Lee HL, Whang S (2004) E-business and supply chain integration. In: The practice of supply chain management: where theory and application converge. Springer, pp 123-138

23. Chen Y, Wang W (2010) Machine-to-machine communication in LTE-A. In: IEEE 72nd vehicular technology conference fall (VTC 2010-Fall). IEEE, pp 1-4

24. ERICSSON Mobility Report on the pulse of the networked society. Technical report, ERICSSON (2014)

25. 3GPP LTE-Advanced. http://www.3gpp.org/technologies/key words-acronyms/97-lte-advanced. Accessed 28 May 2015

26. 3GPP: Technical specification group radio access network; evolved universal terrestrial radio access (E-UTRA); relay architectures for E-UTRA (LTE-Advanced). Technical report, TR 36.806 V9.0.0 (2010)

27. Mehmood Y, Afzal W, Ahmad F, Younas U, Rashid I, Mehmood I (2013) Large scaled multi-user mimo system so called massive mimo systems for future wireless communication networks. In: 2013 19th international conference on automation and computing (ICAC). IEEE, pp 1-4

28. Chandrasekhar V, Andrews JG, Gatherer A (2008) Femtocell networks: a survey. IEEE Commun Mag 46(9):59-67

29. Cox C (2012) An introduction to LTE: LTE, LTE-advanced, SAE and $4 \mathrm{G}$ mobile communications. Wiley, New york

30. METIS: Novel radio link concepts and state of the art analysis. Technical report, Deliverable Number ICT-317669-METIS/D2.2 (2013)

31. Mehmood Y, Marwat SNK, Pötsch T, Ahmad F, Görg C, Rashid I (2014) Impact of M2M traffic on LTE data traffic performance. In: LogDynamics international conference. Springer (in-press)

32. Opnet Modeler Technologies. https://www.opnet.com/services/ modeling-simulation-service.html. Accessed $10 \mathrm{Jul} 2013$

33. Cavers JK (2000) Mobile channel characteristics. Springer, Berlin

34. IEEE 802.16p-11/0014: IEEE 802.16p Machine to Machine (M2M) Evaluation Methodology Document (EMD). Technical report, IEEE 802.16 Broadband Wireless Access Working Group (2010) 